\title{
La Escuela de Artes y Oficios de Santander: un primer esfuerzo por incorporar la educación técnica en la región, 1887-1937
}

The Santander School of Arts and Crafts: A First Effort to Incorporate Technical Education in the Region, 1887-1937

A Escola de Artes e Ofícios de Santander: um primeiro esforço por incorporar a educação técnica na região, 1887 - 1937

\section{Álvaro Acevedo Tarazona* iD orcid.org/0000-0002-3563-9213}

Dayana Lucía Lizcano Herrera** (iD) orcid.org/0000-0003-1651-1887

Edna Lucía Joya Jiménez*** (iD) orcid.org/0000-0003-3267-6710

\footnotetext{
Artículo de investigación

Revista Colombiana de Educación, N.76. Primer semestre de 2019, Bogotá, Colombia.

doi: 10.17227/rce.num76-3082

Para citar este artículo: Acevedo, Á., Lizcano, D. y Joya, E. La Escuela de Artes y Oficios de Santander: un primer esfuerzo por incorporar la educación técnica en la región, 1887-1937. Revista Colombiana de Educación, 76, 173-191.
}

\section{(c) $\underset{\mathrm{BY}(\mathrm{NC})}{(-2)}$}

* Historiador, especialista en Filosofía, magíster en Historia, doctor en Historia, Formación Especializada Docencia en Historia y Cultura de América Latina, magíster a distancia en Historia de América Latina: de la Ilustración al Mundo Contemporáneo, posdoctorado en Ciencias de la Educación. Profesor Universidad Industrial de Santander, Bucaramanga, Colombia. Correo electrónico: acetara@uis.edu.co

** Historiadora y magíster en Historia. Profesora Universidad Santo Tomás Seccional Bucaramanga, Colombia. Correo electrónico: dayana.lizcano@ustabuca.edu.co

*** Historiadora y especialista en Comunicación Social-Periodismo. Profesora Universidad Santo Tomás Seccional Bucaramanga, Colombia. Correo electrónico: edna.joya@ustabuca.edu.co 


\section{Resumen}

La Escuela de Artes y Oficios de Santander fue creada en 1888 con el fin de constituir una fuerza laboral especializada para la realización de los procesos de producción en el departamento. Si bien es importante la contribución de la Escuela para el fomento de la educación técnica y el desarrollo de la industria, son pocos los trabajos historiográficos que existen sobre su trasegar y su impacto. De esta manera, el presente artículo realiza un acercamiento al desenvolvimiento histórico de la institución. Aplicando el método propio de la investigación histórica basado en una sistemática consulta de fuentes primarias y un análisis objetivo de la información, se determinaron las condiciones materiales y educativas que conllevaron a la instauración de la Escuela de Artes y Oficios de Santander, y se identificaron las dinámicas de funcionamiento con el entramado social, político y productivo de dicho departamento. Asimismo, se reconocen aspectos relacionados con los planes de estudio, la oferta de formación, los métodos y prácticas pedagógicas, y sus fuentes de financiación en sus diferentes etapas de existencia. La fundación de este instituto es el primer paso para estudiar el verdadero trasfondo social de esta propuesta educativa.

\section{Palabras clave}

comercio y educación industrial; educación técnica; educación vocacional; industrialización; productividad

\section{Keywords}

Trade and industrial education; technical education; vocational education; industrialization; productivity
The Santander School of Arts and Crafts was created in 1888 with the goal of providing specialized workforce to carry out the production processes in the department. Although the school's contribution to the promotion of technical education and to industrial development is important, there are very few historiographical works about its evolution and impact. As such, this paper addresses the historical development of the institution. By applying the historical research method based on a systematic consultation of primary sources and an objective analysis of the information, we determined the material and educational conditions that led to the establishment of the Santander School of Arts and Crafts and identified the operating dynamics with the social, political and productive context of the Department of Santander. We also identify aspects related to the curricula, the educational offer, the pedagogical methods and practices, and their funding sources in their different stages of existence. The foundation of this institute is the first step to study the true social background of this educational proposal.

\section{Resumo}

A Escola de Artes e Ofícios de Santander foi criada em 1888 com o propósito de constituir uma força de trabalho especializada para a realização dos processos de produção no departamento. Apesar da importância da contribuição da Escola para o fomento da educação técnica e o desenvolvimento da indústria, são poucos os trabalhos historiográficos que existem sobre sua evolução e seu impacto. Dessa forma, este artigo realiza uma aproximação ao desenvolvimento histórico da instituição. Aplicando o método próprio da pesquisa histórica baseado em uma sistemática consulta de fontes primárias e uma análise objetiva da informação, determinamos as condições materiais e educacionais que levaram ao surgimento da Escola de Artes e Oficios de Santander e identificamos as dinâmicas de funcionamento em relação com a estrutura social, política e produtiva desse departamento. Assim mesmo, evidenciamos aspectos relacionados com os planos de estudo, a oferta de formação, os métodos e práticas pedagógicas e suas fontes de financiamento em suas diversas etapas de existência. A fundação deste instituto é o primeiro passo para estudar o verdadeiro contexto social desta proposta educacional.

\section{Palavras-chave}

comércio e educação industrial; educação técnica; educação vocacional; industrialização; produtividade 
En los preludios del siglo xIX, vinculado al nuevo modelo de gobierno liberal con el cual se busca conformar la República en la Nueva Granada, se conciben proyectos educativos que formen individuos en las áreas y con las herramientas requeridas para asumir el compromiso de adelantar el desarrollo de un Estado nacional orientado a la generación de ciudadanos y a la consolidación de los diferentes sectores productivos. Ante la necesidad de formar a la población en favor del mismo Estado, los mentores del proyecto republicano proclaman la enseñanza gratuita, común y uniforme, acorde al destino de una verdadera nación (Codificación, 1924). La estructuración de la educación en sistemas escolarizados se concibe como un requisito para conformar una sociedad acentuada en la libertad, la felicidad, la justicia, la participación y el progreso, distanciada de la organización monárquica española; no obstante, son los vestigios del régimen anterior los que obstaculizan un cambio significativo del modelo educativo durante las primeras décadas del nuevo Gobierno.

Aun así, deben reconocerse las intenciones de generar una organización educativa; razón por la que merecen mencionarse algunos de los propósitos enfocados a la consecución de este fin y reconocer al general Francisco de Paula Santander como un gestor importante del proyecto educativo en los primeros años de la República. El plan de estudios de 1826 representa, de manera oficial, el propósito de convertir a la educación en un mecanismo para la difusión de la moral pública y de conocimientos útiles para el desarrollo económico del país. De esta manera, la educación se distribuye en escuelas de enseñanza primaria, en colegios o casas de educación y en universidades. Las escuelas deberán impartir conocimientos de primer nivel en gramática, religión y aritmética; las escuelas para niñas deberán incorporar áreas que procuren educar en actividades propias del hogar. A los colegios se les asigna la función de enseñar, con una mayor complejidad, los mismos conocimientos impartidos en las escuelas junto con nociones de dibujo, conocimientos de geometría vinculados a las artes mecánicas y saberes de la agricultura práctica. Por su parte, se dispone que las universidades funcionen tan solo en las ciudades más importantes del país y profundicen en matemáticas, física, historia, química y física experimental (Báez, 2004, pp. 242-245).

Este proyecto implica la obligatoriedad del Estado de apoyar la instrucción pública, la instauración de escuelas, colegios y universidades en las diferentes divisiones territoriales, la inclusión femenina en la educación elemental, la implementación de los certámenes públicos para determinar los adelantos en la formación (García, 2007, pp. 209-315) y la adecuación de los métodos de enseñanza modernos, superando la instrucción de prácticas tradicionales (Báez, 2004, pp. 15-39). Si bien se impulsa la instauración de escuelas de primeras letras y de niveles mayores de formación en las ciencias y las artes, y se promueve una educación sin discriminación sexual, 
económica y racial, la carencia de dineros públicos no permite ampliar la cobertura educativa; el clero, con la colaboración de algunos padres de familia, termina por financiar la instauración y mantenimiento de las escuelas de primeras letras.

Desde los inicios del gobierno republicano es perceptible la intención de infundir una educación técnica que supere la formación en el área jurídica, religiosa o médica y que cree un sentido práctico y útil de la educación para promover disciplina, orden, moralidad y utilidad económica. La visión lucrativa de la educación es un propósito ya manifiesto en las políticas reformistas de los Borbones a finales del periodo del régimen indiano; sin embargo, en ambas oportunidades, la tradición proclive a demeritar los saberes en los que se requieren actividades manuales obstaculiza, entre otras explicaciones, su instauración.

Con la llegada al poder estatal del radicalismo político liberal, los programas de gobierno muestran el propósito de abandonar la función netamente moralizante, el dogmatismo religioso, los métodos escolares autoritarios y la poca tendencia a la formación práctica de la educación. De nuevo, la educación se perfila como el principal medio para la transformación social y la conformación del cuerpo político nacional, puesto que permite la formación de personas con un sentimiento nacional y el apoyo a principios republicanos con una base educativa para gestionar desarrollos científicos y tecnológicos conducentes al progreso material del país. La reforma educativa de 1870 es el intento por hacer viable lo concebido y proyectado, entre otras, por su interés de romper definitivamente con las prácticas tradicionales de la enseñanza. Por su parte, la secularización de la educación y la separación de la Iglesia y el Estado se buscan mediante la expulsión de las comunidades religiosas, la expropiación de sus bienes y la inspección de cultos.

Si bien es cierto que la reforma escolar abanderada por el liberalismo radical no sobrevive muchos años y un sinnúmero de sus propuestas no traspasan el plano programático, puesto que la autonomía administrativa con la que cuentan los estados imposibilita que exista uniformidad en las reformas educativas, se logra avanzar en la conversión de la escuela en un instrumento de integración nacional y de formación de ciudadanos, en la promoción de una formación laica y en estimular la promoción de la educación científica y técnica bajo cánones seculares y modernos. Una prueba más de ello es el impulso a la Universidad Nacional; aun así, los cambios más significativos se dan desde el año de 1886 con la conformación oficial de un gobierno centralista y católico conocido como la Regeneración.

Los principios conservadores que sustentan el nuevo régimen de gobierno continúan concibiendo la prioridad de implementar un sistema educativo con diversas modalidades de formación que abarque mayores 
áreas del saber, al igual que el número de personas que puedan beneficiarse de este, puesto que es el mejor mecanismo para alcanzar el anhelado "orden y progreso". Bajo estas premuras, la formación técnica o en campos prácticos halla los escenarios para constituirse, aun cuando encuentra resistencia por parte de algunos sectores sociales que la vinculan con actividades propias de las clases bajas y optan por el estudio de la jurisprudencia, la medicina o las ciencias políticas, entre otras.

En su libro El ideal de lo práctico, Frank Safford denomina a esta dificultad "el proyectismo neoborbónico" (1989). El Gobierno promueve la enseñanza de actividades manuales, el desarrollo industrial y empresarial, la formación ciudadana y los valores cristianos; también impulsa la creación de las escuelas de artes y oficios sustentadas en principios cristianos; ejemplo de esto es la escuela fundada en Santander para el año de 1888. Entre las principales razones que justifican su conformación está la necesidad de formar personas trabajadoras, honradas y dignas, y de impulsar sectores productivos para el crecimiento económico del país (Silva, 1989). El Consejo Nacional Legislativo faculta al Gobierno para crear escuelas de artes y oficios en tres departamentos, una de ellas se instaura en Bucaramanga, la capital del actual departamento de Santander (Ley 121, julio 11 de 1887) con las intenciones de dotar a esta región de un instituto de formación técnica destinado a instruir en conocimientos teóricos y prácticos de las artes y los oficios; confiriendo al personal capacitado grados de maestro, oficial y obrero; con ellas se busca optimizar el trabajo de los obreros, al igual que mejorar la calidad y la presentación de los productos; dentro de estos establecimientos se empiezan a formar artesanos calificados cada vez con mayores bases conceptuales y científicas, capaces de responder a la creciente complejidad técnica de la producción (Gómez, 1995).

Si bien la norma legislativa autoriza al Gobierno para fundar escuelas de artes y oficios hasta en tres de los departamentos de la República, no se especifican las razones para dotar a Bucaramanga de este centro de enseñanza; no obstante, la importancia del departamento de Santander en cuanto a la producción textil puede ser una de ellas. Santander está ubicado en el nororiente de Colombia y ha sufrido diversas transformaciones denominativas de acuerdo con los periodos administrativos por los que ha pasado la nación: a partir de 1857 se denomina Estado Soberano de Santander, conformado por las provincias de Pamplona, Socorro, Vélez y Ocaña. Con el ascenso al poder del presidente Rafael Núñez y con un proceso de centralización, el Estado Soberano de Santander pasó a ser conocido como departamento de Santander, legalización que se dio en 1910 cuando se separaron las provincias de Pamplona, Cúcuta y Ocaña para integrar el departamento de Norte de Santander con capital en Cúcuta, y el actual departamento de Santander quedó constituido por las provincias de Vélez, Comuneros, Guanentá, García Rovira y Soto, con Bucaramanga como capital. 
Para comprender el desarrollo de la instrucción técnica o industrial en el país es importante remitirse al desenvolvimiento institucional educativo de Santander, una de las principales unidades geopolíticas y regionales de la naciente República en gestar establecimientos educativos para formar ciudadanos y dar respuesta a las demandas de la naciente industria nacional. Si bien el impacto de la industrialización en Santander no logra tener la misma importancia que en Antioquia durante la segunda mitad del siglo xIx y el tránsito al siglo xx, merece ser reconocido el trabajo liderado por políticas de gobierno y personajes de la élite en lo que se refiere a políticas de educación, acentuadas en la secularización y en la configuración de instituciones destinadas a la formación para direccionar el progreso y la industrialización. Santander es pionera en la instrucción ciudadana y técnica, de estas iniciativas nacen proyectos educativos relevantes para el departamento y el país; de ahí que este artículo tenga como finalidad reconocer el origen y el desenvolvimiento histórico de la Escuela de Artes y Oficios de Santander, primera institución educativa instaurada en la región para iniciar la formación técnica. Aun cuando no existe una definición unánime e institucional de la educación técnica, de acuerdo con la discursiva que la promueve, esta puede considerarse, para el periodo a estudiar, como una educación para el trabajo basada en conocimientos teóricos y prácticos vinculados a los procesos de producción industrial.

En cuanto a la metodología empleada para efectuar la investigación, soporte del presente texto, es necesario precisar que se acude a la heurística y la hermenéutica, métodos necesarios para la realización de estudios históricos. A través de los principios hermenéuticos se localiza, organiza y evalúa la calidad del aporte de la información contenida en las diferentes fuentes documentales, en su mayoría primarias, como actos legislativos nacionales y departamentales, reglamentación institucional y periódicos oficiales y propios del centro educativo; sin embargo, es importante la contribución de las fuentes secundarias para contextualizar, conceptualizar y apoyar la construcción histórica que desde la crítica de la fuente primaria se logra. La hermenéutica, como método de interpretación, se emplea para el análisis crítico de toda la información recolectada con el fin de alcanzar un mejor acercamiento al hecho histórico abordado.

Si bien se propone identificar las dinámicas de funcionamiento de la Escuela de Artes y Oficios de Santander desde su instauración en el año de 1888 hasta su transición a instituto industrial en 1937, y vincular su hacer a la intención estatal de incorporar la educación técnica, es necesario rastrear los antecedentes del sistema educativo nacional para comprender el marco oficial que concibe la fundación de estos centros de formación, al igual que el entorno social, político y productivo de 
Santander, pues desde allí se reconocen los factores que posibilitan o condicionan el desarrollo de la escuela y el cumplimiento de sus propósitos; de esta manera, se estructura la narrativa del acontecer histórico que presenta este artículo.

\section{La Escuela de Artes y Oficios de Santander: mecanismo educativo para incrementar la productividad y mantener el orden moral}

A imitación de la tradición europea, la modalidad educativa que se adecua
en el periodo colonial es la instrucción doméstica, con la particularidad de
extenderse a familias iletradas o pobres. Sin distanciarse de la enseñanza
impartida en las escuelas de primeras letras, el padre de familia costea la
formación elemental de sus hijos dentro del hogar; los sacerdotes asumen,
en gran parte, esta labor. En menor medida, las familias iletradas, en especial
las que pertenecen a gremios como el de artesanos, se inclinan por vincular
las prácticas educativas al aprendizaje de un oficio específico; así comple-
mentan la formación escolar con una formación para el trabajo. El desprecio
por los oficios "viles" o manuales conduce a que muy pocas familias opten
por hacer que sus hijos los aprendan.

Durante los siglos XVIII y xIx algunas provincias del actual departamento de Santander son modelo de una educación doméstica familiar dirigida a los oficios y el trabajo artesanal y agrícola. La ciudad de Vélez adquiere cierta importancia con sus cultivos de caña de azúcar y la creación de lo que hoy podría llamarse pequeñas empresas de carácter predominantemente familiar. Además del aguardiente, se fortalece la producción textil y de conservas (mieles y dulces) debido a la relativa abundancia de árboles frutales (guayaba, naranja y membrillo) en la región de Vélez. La zona que se extiende al norte de la ciudad de Vélez hasta el Cañón del Chicamocha, abundante en algodón, fique y fibras de paja, se especializa en la producción de confecciones. Alpargatas, mantas, ropas tejidas y sombreros de fibra de jipijapa sostienen económicamente a cientos de familias de las provincias comunera y guanentina; esta producción se presenta como una alternativa económica digna de imitar.

Aun así, en esta región, al igual que en el resto de la Nueva Granada, no se concreta la institucionalidad educativa y menos la escolaridad de los saberes que requieren los trabajos manuales. Esta orientación suscita fuertes críticas por parte de los criollos opositores al régimen monárquico e incluso es uno los cambios que estos plantean en los preludios del proceso independentista. No obstante, la falta de recursos económicos y de una política educativa impulsada y apoyada fuertemente por el Gobierno nacional conlleva a que las pocas instituciones de formación para el trabajo, en 
su mayoría confesionales o religiosas, no cuenten con un notorio respaldo social ni tengan como finalidad principal encausar la capacitación del capital humano a la modernización de la producción.

En el Estado Soberano de Santander, instituido oficialmente en el año de 1857, se identifican propósitos de transformación; a partir de ese momento, el predominio del ideario liberal intenta reorientar la educación, al menos desde el ámbito discursivo. Para el año de 1876, se propone conformar un colegio universitario y anexo a este una escuela normal de artes y oficios (Ley 1, 25 de septiembre de 1876), instituciones educativas sobre las que se legisla en repetidas ocasiones, pero que no llegan a materializarse por la insolvencia económica, los constantes conflictos civiles y la necesidad de priorizar factores constitutivos del Estado.

Durante la experiencia federal de Santander, la educación experimenta dos etapas de orientación. La primera de ellas se presenta entre los años de 1867 y 1876, y se identifica por la tendencia del liberalismo radical en priorizar una formación para el ejercicio de los deberes cívicos y separada de la Iglesia; también se impulsan las escuelas primarias y normales. La segunda etapa, entre los años de 1878 y 1880, se caracteriza por regresar a los principios religiosos como bandera de la educación; para tal efecto, se suprimen las publicaciones oficiales de instrucción pública, aunque se continúa apoyando el método de enseñanza pestalociano propuesto por el liberalismo (Loaiza, 2007, p. 69).

Es en las últimas décadas del siglo xIx, con la conformación de Escuelas de Artes y Oficios que se concretan las intenciones de organizar los programas de formación para el trabajo industrial (Safford, 1989, pp. 95-110). La educación se presenta como un mecanismo para afrontar los problemas por los que franquea el país; tras su fortalecimiento, la educación técnica encuentra condiciones para instituirse. Si bien la enseñanza de actividades manuales mediante un sistema escolarizado contribuye al desarrollo industrial y empresarial, el Gobierno le otorga también otras funciones, como es formar ciudadanos y promover valores cristianos; finalidad que permite entender el sentido de las clases de religión y urbanidad dentro de los planes de estudio. De esta manera, las Escuelas de Artes y Oficios con orientación religiosa son la alternativa para contar con un capital humano formado bajo los principios de la laboriosidad y la honestidad que contribuya a aumentar la productividad económica del país y la generación de una mejor nación (Silva, 1989, p. 80).

Probablemente una de las razones para crear la Escuela de Artes y Oficios es la necesidad de preparar mano de obra calificada que impulse a las incipientes industrias regionales como la textil. La producción textil es una tradición en el departamento de Santander que se remonta a las épocas prehispánicas cuando los primeros pobladores del territorio, los 
indios Guane, fabrican mantas a partir del fique y el algodón que procesan con sus propias manos. Tiempo después, a finales del siglo xvIII y a lo largo de todo el siglo xIX, la producción textil se incrementa con la incorporación de ruecas, telares y husos. La Escuela de Artes y Oficios repercute en la consolidación de la producción, pues tras los cursos impartidos, se fundan pequeños talleres que se constituirán en la base del desarrollo de la industria textil en Santander, permitiendo el emprendimiento de proyectos productivos de interés común.

Durante los siglos XVIII y xIx algunas provincias del actual departamento de Santander se constituyen en modelo de una educación doméstica familiar dirigida a los oficios y el trabajo artesanal y agrícola. La ciudad de Vélez adquiere cierta importancia con la creación de lo que hoy podría llamarse pequeñas empresas de carácter predominantemente familiar. De esta manera se fortalece la producción textil, pues la zona que se extiende al norte de la ciudad de Vélez hasta el Cañón del Chicamocha, abundante en algodón, fique y fibras de paja, se especializa en la producción de confecciones. Alpargatas, mantas, ropas tejidas y sombreros de fibra de jipijapa sostienen económicamente a cientos de familias de las provincias comunera y guanentina. No hay un solo gobernante del Nuevo Reino de Granada que no se refiera a esta producción como una alternativa económica digna de imitar.

A comienzos de 1887, se faculta al Gobierno para crear un máximo de tres escuelas de artes y oficios en toda la geografía nacional, una de ellas se funda en Bucaramanga, capital del departamento de Santander (Ley 121, julio 11 de 1887). Con esta se concretan los propósitos de anteriores Gobiernos por permitir a la región contar con una institución que, desde la enseñanza teórica y práctica, confiriera grados de maestro, oficial y obrero. De acuerdo con el Decreto Orgánico de 1890, la titulación se condiciona a la aprobación de las asignaturas cursadas y de un examen acumulativo y a la presentación de un trabajo práctico de autoría propia (Mayor, 2016, p. 451).

Siguiendo pautas y finalidades del modelo europeo de escuelas de artes y oficios, en especial el francés, Colombia orienta el suyo, condicionado a particularidades del contexto. Culminando el siglo XVIII, algunos países de Europa ven en estos planteles educativos una mejor manera de impulsar la industria, puesto que masifica y diversifica su enseñanza; además, propende por una mejor calidad y presentación de su producción (Pereira y Sousa, 2014). El alcance de sus objetivos alienta las esperanzas y justificaciones para su constitución en este país, el cual requiere con prontitud "artesanos calificados cada vez con mayores bases conceptuales y científicas, capaces de responder a la creciente complejidad técnica de la producción" (Gómez, 1995, p. 16). 
Para abril de 1888, se da apertura a la Escuela de Artes y Oficios de Santander. En el discurso inaugural se deja clara su finalidad de aportar al desarrollo de la clase obrera y de la industria regional; se condiciona el ingreso a cursar los estudios a la residencia de los aspirantes (Pinzón, 1988, pp. 36-39). La creación de la escuela se justifica, de forma reiterada, en la necesidad de proporcionar felicidad nacional, la cual depende de la capacidad de garantizar instrucción al pueblo, ya que este hecho genera bienestar, riqueza y prosperidad; la instrucción se concibe como el alimento del alma, el principio útil de las sociedades y el cerebro de la civilización (El Industrial, junio de 1892, pp. 364-365).

Pasada una década desde que empieza a funcionar, las autoridades departamentales expresan su satisfacción con la Escuela de Artes por "despertar en los jóvenes vocación para algún oficio, y darles los principios y las enseñanzas teóricas y prácticas que los guiarían en el ejercicio de su profesión" (Decreto de enero 15 de 1890). El principio de enseñanza promulgado por sus dirigentes se centra en "enseñar bien la manera de hacer, dejando la rapidez de ejecución que se requiere de un buen obrero, para que el aprendiz la adquiera en la práctica" (El Industrial, julio de 1893, p. 675). En 1892, uno de sus primeros egresados agradece a la institución por enseñarle un oficio mecánico que lo ha dotado de un capital inagotable que lo ayuda en su crecimiento individual y lo hace útil para el beneficio de su sociedad; se reconoce a la escuela el desarrollo de habilidades para la ejecución de los campos aprendidos que les permite a sus egresados fundar sus propios negocios (Mayor, 2016, p. 457) o conseguir un empleo con un buen salario (El Industrial, agosto de 1893, p. 695).

Inicialmente, se estipula que el tiempo para capacitar al estudiantado debe ser de cuatro años, pero la experiencia demuestra que, si se enfatiza en la enseñanza práctica, tres años serán suficientes. Durante los primeros años, las artes y oficios que se ofertan son carpintería, guarnicionería, herrería, zapatería, sastrería y tipografía. El aprendizaje de estas especialidades se complementa con saberes en las siguientes áreas: lectura, literatura, escritura, aritmética, gramática, dibujo, física, geografía, historia, religión y urbanidad (Florencio, 1963, p. 18). Con el tiempo, se suprimen algunas y se anexan otras, entre ellas metalurgia, zoología, gimnasia, música, agronomía y agricultura (Ordenanza 52 de agosto 9 de 1892). Las dos últimas asignaturas se sustentan en la necesidad de formar agricultores hábiles, instruidos y capaces de levantar este sector, como sucede en los países europeos (Ordenanza 13 de agosto 4 de 1890). Siguiendo el ejemplo de escuelas europeas y norteamericanas, se argumenta que la Escuela de Artes y Oficios de Santander debe impartir conocimientos propios de las escuelas de instrucción primaria y secundaria para proporcionar a sus estudiantes mayores herramientas para la obtención de su sustento; sin embargo, este modelo de formación tiene 
detractores entre las personas que están a cargo de la dirección de la institución, ya que consideran que los obreros saldrán mejor capacitados cuando se les brinde una educación exclusiva en un arte u oficio ( $E I$ Industrial, agosto de 1893, p. 676).

Con prontitud, la Escuela de Artes y Oficios vislumbra un mejor porvenir financiero y social para el departamento; así lo manifiesta su gobernador, Guillermo Quintero Calderón, al reconocer su labor en la cualificación de la clase obrera, cada vez con mayor preparación práctica y con fundamentos morales para el crecimiento industrial, el desarrollo individual y una convivencia más pacífica (Florencio, 1963, p. 29); de allí que las cátedras de literatura, urbanidad y religión siempre se incorporan a los diferentes programas de formación que este establecimiento imparte.

En cuanto a su funcionamiento, se debe agregar que la jornada académica inicia a las seis de la mañana y termina por lo general a las ocho y treinta de la noche. Hasta las diez de la mañana se imparten las clases, de once a cuatro de la tarde se orientan los talleres y a las cinco nuevamente se retoman los estudios teóricos hasta finalizar la jornada; entre las seis y siete de la noche, el estudiantado recibe el último alimento del día. Considerando la magnitud de tiempo que los jóvenes deben pasar dentro de la institución y que varios de ellos provienen de otras provincias, la mayoría de estudiantes opta por residir en el plantel; estos deben despertarse a las cinco de la mañana para alcanzar a asearse y a las nueve de la noche deben ir a dormir. El ciclo de actividades para los internos incluye los domingos y días festivos porque están obligados a asistir junto a sus superiores a las celebraciones religiosas (Reglamento de la Escuela de Artes y Oficios, 1890, arts. 66 y 70).

El personal que labora en el plantel está conformado por un director, un secretario, cuatro maestros de artes y varios catedráticos; realmente, el número de profesores lo determina la demanda de estudiantes y los cursos establecidos (Ley 14 de enero 4 de 1887, arts. 5 y7). Para garantizar la calidad de la educación se busca que los docentes sean personas reconocidas por el dominio del área que enseñan y por poseer valores católicos. El Gobierno central faculta al secretario de Instrucción Pública para que cuando lo crea conveniente vincule docentes oriundos de otros departamentos o extranjeros; se conoce que maestros ingleses y españoles apoyan los talleres de herrería y carpintería (Mayor, 2016, pp. 448-450).

Desde su constitución oficial, la Escuela de Artes y Oficios de Santander adquiere un carácter público, la educación debe ser gratuita y los gastos que demanda su funcionamiento serán cancelados con el presupuesto departamental (Ley 121 de julio 11 de 1887, art. 2). No obstante, la falta de liquidez del departamento obliga a sus directivas a gestionar recursos ante el Gobierno nacional y reconocidas personalidades del municipio 
(Pinzón, 1988, p. 48), quienes dan la mayoría de los aportes en especie, ejemplo de esto es la donación de dos máquinas por parte del danés Cristian Peter Clausen (El Industrial, junio de 1892, p. 363). Otra fuente importante de dinero para el sostenimiento de la institución proviene de la comercialización de los productos que allí se fabrican como calzado, fluses, sotanas, capas, candados, cadenas, hachas, hachuelas, sillas y bancas para las iglesias, entre otros (El Industrial, julio de 1892, p. 391); como lo plantea Alberto Mayor, la escuela funciona como un verdadero taller manufacturero, con productos destinados al consumo popular (Mayor, 2016, p. 469). Ante un limitado presupuesto, la formación gratuita para todo el estudiantado debe sustituirse por la asignación de un porcentaje de becas a la población más pobre (Ordenanza 13 de agosto 4 de 1890); según informes internos, el número de becados representa la mitad del total de estudiantes con los que cuenta la escuela, cifra que desciende de acuerdo con el presupuesto departamental.

Sobrellevando las limitantes económicas, la escuela es una importante opción educativa; la pertinencia del conocimiento impartido y la rigurosidad en los métodos de enseñanza incrementan el estudiantado y su reconocimiento, labor en la que también contribuye el periódico institucional, de circulación mensual, titulado El Industrial. El periódico tiene como finalidad informar el desarrollo de la institución y fomentar las artes e industrias en el departamento mediante manuales de procesos productivos; por esta razón, sus destinatarios son artesanos alfabetos y la mayoría dueños de talleres. Considerando la intención de la publicación que se edita en la imprenta del plantel educativo, su obtención no tiene ningún costo (Pinzón, 1988, p. 43); para su obtención debe realizarse una suscripción con los prefectos provinciales, a quienes se los envía el secretario de Gobierno, funcionario encargado de su distribución.

Dados los propósitos económicos y sociales puestos en la Escuela de Artes y Oficios que funciona en Bucaramanga, el Gobierno departamental busca replicar este proyecto en otras localidades como Socorro, San Gil y Pamplona. En el primer municipio debe constituirse un establecimiento educativo exactamente igual al de la capital del departamento (Ordenanza 35 de julio 25 de 1888), en los dos municipios restantes, las escuelas funcionarán anexas a los colegios provinciales San José de Pamplona y San José de Guanentá (Ordenanza 38 de agosto 1. ${ }^{\circ}$ de 1888). Estas últimas adquieren la denominación de "escuela-taller", pero también son afectadas por el bajo presupuesto para su funcionamiento. La escuela de Santander debe cerrarse entre los años de 1894 y 1896 y se denuncian públicamente en el periódico institucional el deterioro en que se encuentran algunas máquinas y herramientas de los diferentes talleres, y la pérdida de otras; así como, el menoscabo de las instalaciones, factores que dificultan el desarrollo de las actividades prácticas (El Industrial, abril de 1896, p. 129). 
Si bien la insolvencia económica es una gran limitante para el cumplimiento del proyecto educativo que representa la Escuela de Artes y Oficios de Bucaramanga y las escuelas-taller del departamento, las continuas guerras civiles repercuten en el normal funcionamiento de estas instituciones y provocan en algunos momentos la suspensión total de sus actividades. Una de ellas se vive en el año de 1899, conocida como la guerra de los Mil Días, se libra fuertemente en Santander y genera el cese indefinido de las clases en todas las instituciones educativas de la región, incluida la Escuela de Artes y Oficios, la cual no puede reabrir al culminar el enfrentamiento armado, puesto que la crisis financiera es cada vez más fuerte.

\section{Después de la guerra, un nuevo impulso para la educación técnica}

La necesidad de continuar la formación de la clase obrera para impulsar el sector industrial conlleva a la reapertura de la Escuela de Artes y Oficios de Santander a comienzos del año de 1912. Se dispone que la institución funcione igual como lo hacía hasta el año de 1899, con las mismas políticas, asignaturas, personal administrativo y docente, instalaciones y cobertura. Con su reapertura, el Gobierno permite a los artesanos y obreros de la región asistir a clases prácticas en el día y durante las noches a las teóricas; este propósito existe desde el año de 1898, pero el cierre impidió su aplicación (Ordenanza 1 de marzo 21 de 1911). La jornada nocturna funciona de seis a nueve de la noche y se organiza en cinco periodos académicos: tres de estudios teóricos y dos de prácticas en talleres.

Otro aspecto nuevo que se incorpora a la normativa de la escuela es la selección de profesores y maestros por concurso público organizado por el director de Instrucción Pública. De nuevo, son muchas las expectativas puestas en la Escuela de Artes y Oficios, pero el exiguo presupuesto departamental impide en repetidas ocasiones el normal funcionamiento de la institución, lo que genera nuevamente su cierre en los años de 1922 y 1929. Además, la inconstancia e inestabilidad de las personas que asisten a la jornada nocturna dificulta la continuidad de esta iniciativa.

Reconociendo los diferentes aportes de la Escuela de Artes y Oficios, la Asamblea del departamento de Santander manifiesta continuamente el interés por reestructurarla, decisión respaldada por el gobernador; sin embargo, a finales del año de 1922, vuelve a clausurarse, estado en el que estará por tres años. El 1. ${ }^{\circ}$ de abril de 1925 , se reinician las clases con un promedio de setenta y cinco estudiantes; con el ánimo de mantener activa la institución, se adhieren a los estudios tradicionales cursos de pintura y una especialización en ayudante de ingeniero (Mayor, 2016, pp. 448-450). 
Para el año de 1929, las clases se interrumpen por causas económicas y por el descenso de estudiantes; durante ese año, se estima que un $40 \%$ de los alumnos abandonan el plantel por expulsión o de manera voluntaria, aspecto que se atribuye a una mejor dirección, ejemplo de esto es el inicio de actividades académicas en 1927 con 70 estudiantes y la culminación con 31 (Mayor, 2016, p. 468). En estas circunstancias, la asamblea opta por detener las partidas destinadas a solventar los gastos de la escuela, lo que provoca su cierre.

Con la llegada nuevamente de los liberales al Gobierno nacional, para el año de 1930, el entorno nacional promete mejorar, incluido el sistema educativo y, por ende, las instituciones que abanderan proyectos de formación claves para el desarrollo de la población. Ante el escaso progreso material y la iliquidez financiera, se arguye la necesidad de consolidar un sistema educativo que apoye la incorporación tecnológica a los procesos productivos y la modernización de las estructuras económicas. En coherencia con las iniciativas nacionales, el Gobierno departamental de Santander vuelve a apostarle a la apertura y reestructuración de la Escuela de Artes y Oficios. Entre las primeras modificaciones está la de encomendar su dirección a una persona con formación técnica y la búsqueda de pertinencia de los programas con "las exigencias modernas de la industria y la enseñanza" (Ordenanza 34 de abril 29 de 1932).

Culminando el año de 1935, el poder legislativo departamental ordena reemplazar la Escuela de Artes y Oficios de Santander por el Instituto de Artes Manuales, cuyo propósito se direcciona a brindar educación técnica en las especialidades industriales de mecánica y electricidad, carpintería y ebanistería, artes textiles y construcciones (Ordenanza 38 de agosto 1 de 1935). Si bien todos los cursos son promocionados por responder a las necesidades del contexto, es evidente la importancia otorgada a las artes textiles; la complejidad de la especialidad requiere que los estudiantes adquieran conocimientos en hilados, tintorerías y tejidos, para intervenir de forma plena en la transformación del algodón, partiendo de su estado natural.

Los planes de estudios se organizan por periodos de cuatro años, las actividades académicas inician el primer día de febrero y terminan el último día de noviembre; a la lista de asignaturas que deben cursar se incorpora la cátedra de Higiene y Economía. Con la intención de mejorar la calidad de la enseñanza, la Asamblea Departamental asigna 24000 pesos para dotar los talleres de tejidos, mecánica, electricidad industrial y carpintería, y suministrar todos los materiales que los estudiantes puedan necesitar; además, se autoriza al gobernador destinar más fondos a medida que el funcionamiento del instituto lo requiera.

Si bien se confía al director determinar los criterios para seleccionar al estudiantado, se le recomienda tener en cuenta la edad, antecedentes de conducta, estado de salud, conocimientos previos y, en ocasiones, el 
estado económico, ya que el Gobierno departamental insiste en la necesidad de ofrecer gratuitamente la enseñanza técnica. Con la reestructuración de este establecimiento educativo se ordena la contratación, casi exclusiva, de personal santandereano para desempeñar las actividades académicas y administrativas; factor que puede obedecer a la intención de estimular a los maestros del departamento, la mayoría formados en la Escuela de Artes y Oficios, o evitar los grandes gastos que demanda la contratación de extranjeros.

Pese a que el cambio nominal no dura más de un año, porque para 1936 la escuela retoma su primera denominación (Ordenanza 59 de mayo 4 de 1936), desde el deber que contempla la discursiva oficial se identifica la intención de mejorar el espacio locativo de la institución, aunque en gran parte obedezca a la obligatoriedad de cumplir con las exigencias del Departamento Nacional de Higiene (Pinzón, 1988, p. 72).

El menoscabo de la planta física y la falta de presupuesto para edificar nuevas locaciones condicionan el funcionamiento de la escuela y la materialización de la propuesta nacional de impulsar la educación técnica. Como solución, se dispone trasladar y vincular esta institución al Colegio de Santander, primer establecimiento oficial de bachillerato en Bucaramanga (Decreto 630 de agosto 6 de 1936); la imposibilidad de hacer funcionar dentro de una misma infraestructura a dos instituciones con diferente modalidad de formación provoca su desvinculación y, con ella, la nueva denominación de la escuela, que pasa a llamarse escuela industrial (Decreto 453 de mayo 14 de 1937); al poco tiempo, la categoría de escuela es reemplazada por la de instituto, modificación que representa el objetivo de redirigir su labor, apartándola de conocimientos o saberes básicos.

Teniendo en cuenta la importancia de este instituto para el desarrollo material y social de Santander y los problemas de espacio que padece para el cumplimiento de sus funciones, los hermanos Navas Serrano ceden un lote en la parte norte de la ciudad para la construcción de un edificio (Pinzón, 1988, p. 73). Sin embargo, la carencia de dinero para adelantar la obra, retarda los planes; el Gobierno nacional debe asumir gran parte de su costo, el departamento solo puede contribuir con 10000 pesos (Ordenanza 50 de junio 28 de 1940). Con la nueva infraestructura se propone "incrementar las secciones de estudio, mejorar la organización y crear secciones superiores de enseñanza para la preparación de técnicos" (Pinzón, 1988, p. 73). En un pronunciamiento público, el director de Educación de Santander, Horacio Rodríguez Plata, destaca la labor de conceder al instituto una apropiada edificación, puesto que su función se encamina a impulsar la industria regional y activar la economía local. El disponer de mejores espacios físicos motiva el propósito de incorporar otros niveles de formación vinculados al que ya tiene el instituto.

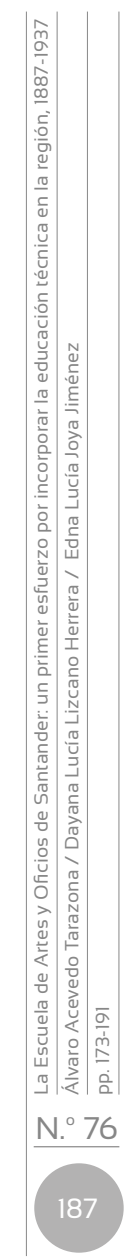


Durante la Asamblea de Directores de Educación, realizada en la ciudad de Bogotá en el año de 1940, se dispone la reorganización de la enseñanza vocacional, con gran preferencia por el bachillerato industrial, dado que se concibe que una de las maneras de estimular la industria nacional es formar cuadros de técnicos; es decir, la formación consecutiva de todos los niveles de educación técnica, obreros auxiliares, obreros calificados, expertos, técnicos e ingenieros. Las conclusiones de la asamblea son transmitidas a la Dirección de Educación del departamento por el ingeniero español Julio Álvarez Cerón, quien argumenta la necesidad de contar con planes educativos escalonados (Lizcano, 2012); de allí su labor en la instauración de la Facultad de Ingeniería Industrial, orientada a las especializaciones de Química, Mecánica y Electricidad, la cual representa la primera institución de educación superior en la región, la Universidad Industrial de Santander (Ordenanza 41 de junio 21 de 1940).

La instauración del bachillerato técnico se sustenta en la necesidad de brindar a los jóvenes un saber científico y de la experiencia práctica para efectuar trabajos en el amplio campo de la producción; a las universidades, como la Industrial de Santander, se les otorga la función de formar en conocimientos técnicos y científicos. Con la implementación de este proyecto educativo, el departamento podrá gozar de cuadros completos de expertos, técnicos e ingenieros industriales en diversas especialidades (Álvarez, 2008, pp. 12-17).

Por su parte, es hasta el año de 1944 que el instituto industrial puede estrenar su planta física, y con ella su nueva denominación: Instituto Industrial Dámaso Zapata (Decreto 1427 de diciembre 29 de 1941), como reconocimiento al educador santandereano y para dar acatamiento a la Ley 55 de 1924, que dispone llamar de esta manera a un internado en zona rural; sin embargo, su no conformación Ileva a la administración del departamento a adjudicar su nombre al instituto industrial. De acuerdo con la intención de formar cuadros escalonados de técnicos, se realizan modificaciones curriculares para la formación de expertos y bachilleres técnicos. La enseñanza industrial básica concede la titulación de expertos y la profundización en el saber científico y las labores prácticas, y, durante dos años más de estudios, otorga el grado de bachiller técnico. Los primeros bachilleres culminan sus estudios en el año de 1947, los cuales dan apertura a la Universidad Industrial, con ello se cumplen las intenciones de conformar cuadros completos de profesionales técnicos para impulsar la industria nacional. 


\section{Conclusiones}

Desde un comienzo, la educación técnica se concibe como una estrategia para incrementar la productividad y mantener el orden moral. Paradójicamente, el estímulo a la formación para el trabajo industrial, apuesta insigne del radicalismo liberal, llega a desarrollarse y fortalecerse bajo la tutela de la Regeneración; lo cual permite afirmar que el interés por desarrollar una educación técnica y tecnológica no es propiedad de un partido político ni de una ideología. Es así como el propósito de fundar escuelas de artes y oficios se concreta bajo la tutela de un Gobierno centralista y católico, sin perder la intención de formar individuos laboriosos, honestos y dignos, capacitados para impulsar los diferentes sectores productivos y contribuir al crecimiento económico del país. Con estos propósitos se instaura en el año de 1888 la Escuela de Artes y Oficios de Santander, gestora de la instrucción ciudadana y técnica. Si bien, la enseñanza de actividades manuales mediante un sistema escolarizado contribuye al desarrollo industrial y empresarial, el Gobierno departamental le adhiere otras funciones, como es formar ciudadanos y sembrar valores cristianos; razón por la que los planes de estudios son versátiles e incorporan diferentes áreas del saber.

La Escuela de Artes y Oficios de Santander es una institución pública proyectada a brindar educación gratuita, pero la carencia de fondos departamentales conlleva a buscar otras fuentes de financiamiento, entre ellas, auxilios del Gobierno nacional, aportes de particulares y la venta de productos fabricados dentro del plantel educativo; la comercialización de sus productos hace que la escuela llegue a considerarse un verdadero taller manufacturero. Sin embargo, el débil presupuesto departamental y la inestabilidad política obstaculizan su normal funcionamiento, ocasionando su cierre en repetidas ocasiones.

La rigidez en los procesos de enseñanza y el empeño de sus dirigentes permiten que la educación impartida sea de calidad, aspecto que le otorga reconocimiento como centro de formación para el trabajo. Sus egresados son reconocidos por sus iniciativas empresariales y por el buen desempeño de sus labores. En los años treinta, la necesidad de generar una educación con un mayor enfoque técnico, ligada a los procesos industriales de la región, provoca la transformación de la escuela en el Instituto Industrial Dámaso Zapata, el cual se vincula a un proyecto educativo centrado en la instrucción de cuadros completos de formación, es decir, técnicos, tecnólogos e ingenieros. 


\section{Referencias}

\section{Fuentes primarias}

Archivo de la Asamblea del Estado Soberano de Santander, Bucaramanga, 1876, 1890, 1892-1893.

Archivo de la Asamblea de Santander, Bucaramanga, 1888, 1890, 1911, 1932, 1935, 1936, 1940.

Archivo de la Gobernación de Santander, Bucaramanga, 1888, 1890, 1936-1937.

Archivo del Congreso de la República, Bogotá, 1887.

Codificación Nacional, Bogotá, 1924.

Periódico El Industrial, Bucaramanga, 1892-1893, 1896.

Reglamento de la Escuela de Artes y Oficios, Bucaramanga, 1890.

\section{Fuentes secundarias}

Álvarez, J. (2008). La ciencia y la técnica en la universidad, 1940. Revista de Santander, 3, 12-17.

Báez, M. (2004). Las escuelas normales y el cambio educativo en los Estados Unidos de Colombia en el Periodo Radical, 1870-1886. Tunja: Universidad Pedagógica y Tecnológica de Colombia.

Florencio, R. (1963). Historia del Instituto Superior Dámaso Zapata. Bucaramanga: Imprenta del Departamento.

García, B. (2007). De la educación doméstica a la educación pública: transiciones de la Colonia a la República. Bogotá: Universidad Distrital Francisco José de Caldas.

Gómez, V. (1995). La educación tecnológica en Colombia. ¿Educación terminal o primer ciclo de las ingenierías y las ciencias? Bogotá: Universidad Nacional.

Lizcano, D. (2012). Las dinámicas de desarrollo de la educación técnica y tecnológica en Santander mediante un estudio institucional. Temas, 3(6), 73-96.

Loaiza, G. (2007). El maestro de escuela o el ideal liberal de ciudadano en la reforma educativa de 1870. Historia Crítica, (4). Recuperado de https://historiacritica.uniandes.edu.co/view.php/258/1.php.

Mayor, A. (2016). Las escuelas de artes y oficios en Colombia 1860-1960. Bogotá: Pontificia Universidad Javeriana.

Pereira, F. y Sousa, J. B. (2014). El origen de las escuelas de artes y oficios en Galicia. Recuperado de http://campus.usal.es/ revistas_trabajo/ index.php/0212-0267/article/viewFile/6899/6881. 
Pinzón, R. (1988). Por tierras de Santander (el Dámaso Zapata). Bucaramanga: Estudio.

Safford, F. (1989). El ideal de lo práctico: el desafío de formar una élite técnica y empresarial en Colombia. Bogotá: Universidad Nacional-El Áncora.

Silva, R. (1989). La educación en Colombia, 1880-1930. En J. Melo, Nueva historia de Colombia (Iv, pp. 61-86). Bogotá: Planeta. 
